\title{
Unital strongly harmonic commutative Banach algebras
}

\author{
by \\ JANKo BRAČIČ (Ljubljana)
}

\begin{abstract}
A unital commutative Banach algebra $\mathcal{A}$ is spectrally separable if for any two distinct non-zero multiplicative linear functionals $\varphi$ and $\psi$ on it there exist $a$ and $b$ in $\mathcal{A}$ such that $a b=0$ and $\varphi(a) \psi(b) \neq 0$. Spectrally separable algebras are a special subclass of strongly harmonic algebras. We prove that a unital commutative Banach algebra $\mathcal{A}$ is spectrally separable if there are enough elements in $\mathcal{A}$ such that the corresponding multiplication operators on $\mathcal{A}$ have the decomposition property $(\delta)$. On the other hand, if $\mathcal{A}$ is spectrally separable, then for each $a \in \mathcal{A}$ and each Banach left $\mathcal{A}$-module $\mathcal{X}$ the corresponding multiplication operator $L_{a}$ on $\mathcal{X}$ is super-decomposable. These two statements improve an earlier result of Baskakov.
\end{abstract}

1. Introduction. Let $\mathcal{A}$ be a complex commutative Banach algebra and let $\Sigma(\mathcal{A})$ denote the spectrum of $\mathcal{A}$, i.e. the set of all non-zero multiplicative linear functionals on $\mathcal{A}$. The usual (or Gelfand) topology on $\Sigma(\mathcal{A})$ is the relative weak-* topology. It is well known that there is a bijective correspondence between points of $\Sigma(\mathcal{A})$ and maximal modular ideals in $\mathcal{A}$. If $\varphi$ is from $\Sigma(\mathcal{A})$, let $M_{\varphi}(=\operatorname{ker} \varphi)$ be the corresponding ideal.

In [3] Baskakov studied spectral synthesis in Banach modules over spectrally separable algebras. These algebras are defined as follows.

A unital commutative Banach algebra $\mathcal{A}$ is spectrally separable if for any two distinct functionals $\varphi$ and $\psi$ in $\Sigma(\mathcal{A})$ there exist elements $a$ and $b$ in $\mathcal{A}$ such that $a b=0$ and $\varphi(a) \psi(b) \neq 0$.

It can be easily verified that a unital commutative Banach algebra $\mathcal{A}$ is spectrally separable if and only if for each pair $M_{\varphi}, M_{\psi}$ of distinct maximal modular ideals of $\mathcal{A}$, there are ideals $\mathcal{I}$ and $\mathcal{J}$ (not necessarily closed) satisfying $\mathcal{I} \not \subset M_{\varphi}, \mathcal{J} \not \subset M_{\psi}$ and $\mathcal{I} \mathcal{J}=\{0\}$. But this means that $\mathcal{A}$ is spectrally separable if and only if it is a unital commutative Banach algebra that is also strongly harmonic in the sense of [9] (cf. [17], §7.4).

2000 Mathematics Subject Classification: Primary 46J05, 46H25; Secondary 47B40, $47 \mathrm{~B} 48$.

Key words and phrases: Banach module, commutative Banach algebra, decomposition property $(\delta)$, multiplication operator, spectrally separable algebra, strongly harmonic algebra, super-decomposable operator. 
Note that strongly harmonic algebras are a special subclass of harmonic algebras which were introduced by Teleman in [18]. By definition, an algebra $\mathcal{A}$ over a unital ring $\mathcal{R}$ is harmonic if its space of maximal modular ideals, with the hull-kernel topology, is a Hausdorff space. For instance, a unital commutative complex Banach algebra is harmonic if and only if it is regular. There exist harmonic algebras that are not strongly harmonic.

ExAmple 1.1 ([9], Example 2.11). Let $\mathbb{Q}$ be the field of rational numbers and let $p_{1}, \ldots, p_{l}(l \geq 2)$ be a finite number of distinct prime numbers. Denote by $\mathcal{A}$ the set

$$
\left\{m / n \in \mathbb{Q} ; n \text { is not divisible by any } p_{1}, \ldots, p_{l}\right\} .
$$

It is easily seen that $\mathcal{A}$ is a unital algebra over the ring $\mathbb{Z}$ of integers. This algebra is an example of a harmonic algebra which is not strongly harmonic (for the argument see [9]).

However, we are interested only in unital commutative complex Banach algebras. It seems to be an open question whether there exist unital commutative harmonic complex Banach algebras which are not strongly harmonic, i.e. unital commutative complex Banach algebras which are regular but not spectrally separable.

Throughout this paper we will use the term spectrally separable algebra to mean a unital strongly harmonic commutative complex Banach algebra.

Baskakov has shown that all multiplication operators on a spectrally separable algebra are decomposable in the sense of Foias and, on the other hand, that a unital commutative Banach algebra $\mathcal{A}$ is spectrally separable if there are enough decomposable multiplication operators on $\mathcal{A}$ (see [3], Theorem 2). This result extends the work of Colojoară and Foiaş ([5], Theorem 6.2.6) and Frunză ([7], Theorem 2) who proved that a semisimple unital commutative Banach algebra is regular if and only if all multiplication operators are decomposable.

In this article (our main results are in Section 3) we extend Baskakov's result in two directions. In Theorem 3.4 we show that for a spectrally separable algebra $\mathcal{A}$ and a Banach $\mathcal{A}$-module $\mathcal{X}$, each multiplication by an element of $\mathcal{A}$ on $\mathcal{X}$ is a super-decomposable operator in the sense of [11]. On the other hand Theorem 3.5 (see also Theorem 3.7) shows that a unital commutative Banach algebra is spectrally separable if there are enough multiplication operators on it which have the decomposition property $(\delta)$. Our results should be compared with those in [12], [13], [15], [16], and [6]. Note however that our approach makes semisimplicity assumptions in some cases redundant.

In Section 2 we will state some known assertions about spectrally separable algebras and we shall give some examples. In the remainder of this section we introduce the notation. 
If $a$ is an element in a commutative Banach algebra $\mathcal{A}$ then $\widehat{a}: \Sigma(\mathcal{A}) \rightarrow \mathbb{C}$ is its Gelfand transform. We denote by $Z(a):=\{\varphi \in \Sigma(\mathcal{A}) ; \widehat{a}(\varphi)=0\}$ the zero set of $a$ and with $\omega(a)$ the complement of $Z(a)$ in $\Sigma(\mathcal{A})$. The support $\operatorname{supp}(a)$ of $a$ is the closure of $\omega(a)$ in the Gelfand topology.

If $E$ is a subset in $\Sigma(\mathcal{A})$ then the kernel of $E$, denoted by $k(E)$, is defined as

$$
k(E):=\bigcap_{\varphi \in E} \operatorname{ker} \varphi=\{a \in \mathcal{A} ; Z(a) \supseteq E\} .
$$

The hull $h(\mathcal{M})$ of a subset $\mathcal{M}$ in $\mathcal{A}$ is defined as

$$
h(\mathcal{M}):=\{\varphi \in \Sigma(\mathcal{A}) ; \mathcal{M} \subseteq \operatorname{ker} \varphi\}=\bigcap_{a \in \mathcal{M}} Z(a) .
$$

It is well known that the correspondence $E \mapsto h(k(E))$ is a closure operation and that the topology on $\Sigma(\mathcal{A})$ determined by this closure operation, called the hull-kernel topology, is weaker than the Gelfand topology. It follows from the definition that the zero set of $a \in \mathcal{A}$ is hull-kernel closed. Hence $\omega(a)$ is always hull-kernel open. If the Gelfand topology and the hull-kernel topology coincide on $\Sigma(\mathcal{A})$, then the algebra $\mathcal{A}$ is said to be regular. The reader is referred to [10] for details.

Let $\mathcal{A}$ be a commutative Banach algebra and let $\mathcal{X}$ be a Banach space. Then $\mathcal{X}$ is a Banach left $\mathcal{A}$-module if it is a left $\mathcal{A}$-module (for the definition see [4], Definition 11 in $\S 9$ ) and if there is a constant $k$ such that

$$
\|a x\| \leq k\|a\| \cdot\|x\|, \quad a \in \mathcal{A}, x \in \mathcal{X} .
$$

By renorming $\mathcal{X}$ with an equivalent norm, we may suppose that $k=1$. If $\mathcal{A}$ has a unit 1 , then it is assumed that $1 x=x$ for all $x \in \mathcal{X}$.

If $\mathcal{X}$ is a Banach left $\mathcal{A}$-module, then $\mathcal{X}^{*}$, the dual space of $\mathcal{X}$, is the dual $\mathcal{A}$-module of $\mathcal{X}$. The operation is given by $\langle a \xi, x\rangle=\langle\xi$, ax $\rangle$ for $a \in \mathcal{A}$, $\xi \in \mathcal{X}^{*}$ and $x \in \mathcal{X}$.

For a subset $M$ of a Banach left $\mathcal{A}$-module $\mathcal{X}$ let ann ${ }_{\mathcal{A}} M$ denote the annihilator of $M$, that is, the set $\{a \in \mathcal{A} ; a x=0$ for all $x \in M\}$. It is easy to see that $\operatorname{ann}_{\mathcal{A}} M$ is a closed ideal in $\mathcal{A}$. If $M$ is nonempty, then the spectrum $\operatorname{Sp}(M)$ of $M$ is the hull of the annihilator $\operatorname{ann}_{\mathcal{A}} M$, that is, $\operatorname{Sp}(M)=h\left(\operatorname{ann}_{\mathcal{A}} M\right) \subseteq \Sigma(\mathcal{A})$. We set $\operatorname{Sp}(\emptyset)=\emptyset$. When $M$ is a singleton $\{x\}$ we shall write $\operatorname{Sp}(x)$ instead of $\operatorname{Sp}(\{x\})$. Since the algebra $\mathcal{A}$ is a Banach left $\mathcal{A}$-module for the usual multiplication in $\mathcal{A}$, the spectrum may be defined also for subsets of $\mathcal{A}$. It is easy to see that $\operatorname{supp}(a) \subseteq \operatorname{Sp}(a)$ for all $a \in \mathcal{A}$ and we have $\operatorname{supp}(a)=\operatorname{Sp}(a)$ when $\mathcal{A}$ is regular and semisimple.

In the following proposition we list (without proofs) some basic properties of spectra (cf. [3], Lemma 1).

Proposition 1.2. Let $\mathcal{A}$ be a commutative Banach algebra and let $\mathcal{X}$ be a Banach left $\mathcal{A}$-module. 
(i) For any subset $M \subseteq \mathcal{X}$ the spectrum $\operatorname{Sp}(M)$ is a closed subset of $\Sigma(\mathcal{A})$. Hence, if $\mathcal{A}$ has a unit, then $\operatorname{Sp}(M)$ is compact.

(ii) If $\mathcal{A}$ has a unit then the spectrum $\mathrm{Sp}(M)$ of a nonempty subset $M \subseteq \mathcal{X}$ is empty if and only if $M=\{0\}$.

(iii) For every $a \in \mathcal{A}$ and every $x \in \mathcal{X}$ we have the inclusions

$$
h(k(\omega(a) \cap \mathrm{Sp}(x))) \subseteq \mathrm{Sp}(a x) \subseteq \operatorname{Sp}(a) \cap \mathrm{Sp}(x) .
$$

(iv) Let the algebra $\mathcal{A}$ have a unit. If $\operatorname{Sp}(a) \cap \operatorname{Sp}(x)=\emptyset$, then ax $=0$, and if $\operatorname{Sp}(a-1) \cap \operatorname{Sp}(x)=\emptyset$, then $a x=x$.

(v) $\operatorname{Sp}(\mathcal{X})=\operatorname{Sp}\left(\mathcal{X}^{*}\right)$.

(vi) If $x_{1}, \ldots, x_{n} \in \mathcal{X}$ and $x=x_{1}+\ldots+x_{n}$ then $\operatorname{Sp}(x) \subseteq \bigcup_{k=1}^{n} \operatorname{Sp}\left(x_{k}\right)$.

We point out that the spectrum we have intruduced is just another aspect of the well known Arveson spectrum. Namely, let $\mathcal{X}$ be a left Banach $\mathcal{A}$-module and let $\theta: \mathcal{A} \rightarrow B(\mathcal{X})$ be the corresponding representation of the algebra $\mathcal{A}$ on $\mathcal{X}$, that is, $\theta(a) x=a x(a \in \mathcal{A}, x \in \mathcal{X})$. Then $\operatorname{Sp}(\mathcal{X})$ is the Arveson spectrum of the representation $\theta$, and $\operatorname{Sp}(x)$ is the local Arveson spectrum of $\theta$ at $x \in \mathcal{X}$ (cf. Section 4.12 in [14]).

2. Spectrally separable algebras. The results of this section are more or less known (cf. [3] and [17], §7.4). Since some statements are given without proofs in [3], we shall prove them.

Recall that a unital commutative Banach algebra $\mathcal{A}$ is spectrally separable if for any two distinct functionals $\varphi$ and $\psi$ in $\Sigma(\mathcal{A})$ there exist elements $a$ and $b$ in $\mathcal{A}$ such that $a b=0$ and $\widehat{a}(\varphi) \widehat{b}(\psi) \neq 0$. Of course, if the spectrum of $\mathcal{A}$ is empty or finite, then $\mathcal{A}$ is spectrally separable. The next example shows that there are spectrally separable algebras whose spectrum is richer.

Recall from [8] that a topological space $X$ is 0 -dimensional if the family of all sets that are both open and closed is an open basis for the topology of $X$. For example, if $X$ is locally compact, Hausdorff, and totally disconnected, then $X$ is 0 -dimensional ([8], Theorem 3.5). Thus, if the spectrum $\Sigma(\mathcal{A})$ of a commutative Banach algebra $\mathcal{A}$ is totally disconnected, then it is 0 dimensional.

EXAMPLE 2.1. If the spectrum $\Sigma(\mathcal{A})$ of a regular unital commutative Banach algebra $\mathcal{A}$ is 0 -dimensional, then $\mathcal{A}$ is spectrally separable.

Proposition 2.2. Every spectrally separable algebra is regular.

Proof. See [17], Proposition 7.4.2.

Corollary 2.3. Let $\mathcal{A}$ be a unital semisimple commutative Banach algebra. Then $\mathcal{A}$ is regular if and only if $\mathcal{A}$ is spectrally separable.

In the following proposition it is shown that the class of spectrally separable algebras is stable under some operations. Let $\mathcal{A}$ and $\mathcal{B}$ be com- 
mutative Banach algebras. Denote by $\mathcal{A} \widehat{\otimes} \mathcal{B}$ the projective tensor product of $\mathcal{A}$ and $\mathcal{B}$, that is, the completion of the algebraic tensor product $\mathcal{A} \otimes \mathcal{B}=\left\{\sum_{i=1}^{n} a_{i} \otimes b_{i} ; a_{i} \in \mathcal{A}, b_{i} \in \mathcal{B}\right\}$ under the projective cross norm $\|\cdot\|_{\gamma}$, which is defined by

$$
\|u\|_{\gamma}=\inf \left\{\sum_{i=1}^{n}\left\|a_{i}\right\| \cdot\left\|b_{i}\right\| ; u=\sum_{i=1}^{n} a_{i} \otimes b_{i}\right\}
$$

where the infimum is taken over all finite representations of $u$ (cf. [4], §42).

Proposition 2.4 (cf. [3]). (i) If $\mathcal{A}$ is a spectrally separable algebra and $\mathcal{I} \subseteq \mathcal{A}$ is a closed ideal of $\mathcal{A}$, then $\mathcal{A} / \mathcal{I}$ is a spectrally separable algebra.

(ii) If $\mathcal{A}$ and $\mathcal{B}$ are spectrally separable algebras, then so are $\mathcal{A} \times \mathcal{B}$ and $\mathcal{A} \widehat{\otimes} \mathcal{B}$.

(iii) Let $\mathcal{A}$ be a spectrally separable algebra and let $\mathcal{B}$ be a unital commutative Banach algebra. If $\Phi: \mathcal{A} \rightarrow \mathcal{B}$ is a unital homomorphism whose image $\Phi(\mathcal{A})$ is dense in $\mathcal{B}$, then $\mathcal{B}$ is a spectrally separable algebra.

Proof. If we identify $\Sigma(\mathcal{A} / \mathcal{I})$ with the subset $h(\mathcal{I})$ of $\Sigma(\mathcal{A})([10]$, Theorem 7.3.1.), and $\Sigma(\mathcal{A} \times \mathcal{B})$ and $\Sigma(\mathcal{A} \widehat{\otimes} \mathcal{B})$ are identified with the sets $\{(\varphi, 0) ; \varphi \in \Sigma(\mathcal{A})\} \cup\{(0, \psi) ; \psi \in \Sigma(\mathcal{B})\}$ and $\Sigma(\mathcal{A}) \times \Sigma(\mathcal{B})$, respectively (for the last see [4], §43, Proposition 19, p. 236), then (i) and (ii) follow from the definition of a spectrally separable algebra.

(iii) It is standard that for two distinct points $\varphi$ and $\psi$ in $\Sigma(\mathcal{B})$ the functionals $\Phi^{*} \varphi$ and $\Phi^{*} \psi$ are distinct elements in $\Sigma(\mathcal{A})$. Hence, by definition, there are $a$ and $b$ in $\mathcal{A}$ such that $a b=0$ and $\widehat{a}\left(\Phi^{*} \varphi\right) \widehat{b}\left(\Phi^{*} \psi\right) \neq 0$. Now it is easy to see that $\Phi(a)$ and $\Phi(b)$ are such that $\Phi(a) \Phi(b)=0$ and $(\Phi(a))(\varphi)(\Phi(b))(\psi) \neq 0$.

Let $\mathcal{A}$ and $\mathcal{B}$ be spectrally separable algebras. If $\|\cdot\|_{\alpha}$ is a cross norm on $\mathcal{A} \otimes \mathcal{B}$ such that $\mathcal{A} \otimes_{\alpha} \mathcal{B}$ (the closure of $\mathcal{A} \otimes \mathcal{B}$ under the cross norm $\|\cdot\|_{\alpha}$ ) is a Banach algebra, then it is a spectrally separable algebra by (ii) and (iii) of the preceding proposition.

Let $\mathcal{A}$ be a commutative Banach algebra and suppose $E \subseteq \Sigma(\mathcal{A})$ is closed. Then $j_{0}(E)$ will denote the set of all $a \in \mathcal{A}$ for which $\widehat{a}$ vanishes identically on some open neighbourhood of $E$ and the $\operatorname{support} \operatorname{supp}(a)$ is a compact subset of $\Sigma(\mathcal{A})$. Let $j(E)$ be the closure of $j_{0}(E)$. If $\mathcal{A}$ is regular then the hull of $j(E)$ is $E$ and if $\mathcal{A}$ is also semisimple then $j(E)$ is the smallest closed ideal in $\mathcal{A}$ whose hull is $E$ ([10], Theorem 8.1.1).

Let $\mathcal{A}$ be a commutative semisimple regular Banach algebra. Then a closed set $E \subseteq \Sigma(\mathcal{A})$ is said to be a set of spectral synthesis if $j(E)=k(E)$. Thus, if $E$ is a set of spectral synthesis, then the kernel $k(E)$ is the unique closed ideal in $\mathcal{A}$ whose hull is $E$. The closed sets in $\Sigma(\mathcal{A})$ which are not sets of spectral synthesis are sets of spectral non-synthesis. It is well known that 
there are semisimple regular algebras with sets of spectral non-synthesis (cf. [10], §8.2 and $\S 8.3)$.

ExAMPLE 2.5. Let $\mathcal{A}$ be a unital semisimple regular commutative Banach algebra and let $E \subseteq \Sigma(\mathcal{A})$ be a set of spectral non-synthesis. Then $\mathcal{A} / j(E)$ is a spectrally separable algebra which is not semisimple.

LEMma 2.6. Let $\mathcal{A}$ be a unital commutative Banach algebra. Then $\mathcal{A}$ is spectrally separable if and only if for every compact subset $F \subset \Sigma(\mathcal{A})$ and every $\varphi \in \Sigma(\mathcal{A}) \backslash F$ there exists an element $a \in \mathcal{A}$ such that $\widehat{a}(\varphi)=1$ and $\operatorname{Sp}(a) \cap F=\emptyset$.

Proof. Assume first that $\mathcal{A}$ is spectrally separable. Let $F \subset \Sigma(\mathcal{A})$ be compact and let $\varphi \in \Sigma(\mathcal{A}) \backslash F$. By definition, there are $a_{\psi}$ and $b_{\psi}$ in $\mathcal{A}$ such that $a_{\psi} b_{\psi}=0$ and $\widehat{a}_{\psi}(\varphi) \widehat{b}_{\psi}(\psi) \neq 0$ for every $\psi \in F$. It follows that $\operatorname{Sp}\left(a_{\psi}\right) \cap \omega\left(b_{\psi}\right)=\emptyset$ by Proposition 1.2. The family $\left\{\omega\left(b_{\psi}\right) ; \psi \in F\right\}$ is an open covering of $F$. Thus, there are $\psi_{1}, \ldots, \psi_{n}$ in $F$ such that $F \subset \omega\left(b_{\psi_{1}}\right) \cup$ $\ldots \cup \omega\left(b_{\psi_{n}}\right)=: U$. Define $a^{\prime}:=a_{\psi_{1}} \ldots a_{\psi_{n}}$. The spectrum $\operatorname{Sp}\left(a^{\prime}\right)$ is included in $\operatorname{Sp}\left(a_{\psi_{1}}\right) \cap \ldots \cap \operatorname{Sp}\left(a_{\psi_{n}}\right)$, therefore $\operatorname{Sp}\left(a^{\prime}\right) \cap U=\emptyset$. The number $\widehat{a^{\prime}}(\varphi)=$ $\widehat{a}_{\psi_{1}}(\varphi) \ldots \widehat{a}_{\psi_{n}}(\varphi)$ is not zero, hence for $a=\widehat{a}^{\prime}(\varphi)^{-1} a^{\prime}$ we have $\widehat{a}(\varphi)=1$ and $\operatorname{Sp}(a) \cap U=\emptyset$.

Let $\varphi$ and $\psi$ be two distinct functionals in $\Sigma(\mathcal{A})$. Since $\Sigma(\mathcal{A})$ is compact and Hausdorff there is an open neighbourhood $U$ of $\varphi$ such that $\bar{U}^{\mathrm{c}}$ is an open neighbourhood of $\psi$. The sets $\bar{U}$ and $U^{\mathrm{c}}$ are compact, hence there are $a$ and $b$ in $\mathcal{A}$ such that $\widehat{a}(\varphi)=1, \widehat{b}(\psi)=1, \operatorname{Sp}(a) \cap U^{\mathrm{c}}=\emptyset$, and $\operatorname{Sp}(b) \cap \bar{U}=\emptyset$. Thus, we have $\operatorname{Sp}(a) \cap \operatorname{Sp}(b)=\emptyset$ and, by Proposition $1.2, a b=0$. This proves the opposite direction.

Let $\mathcal{A}$ be a spectrally separable algebra and let $\mathcal{X}$ be a Banach left $\mathcal{A}$-module. For any subset $S$ of $\Sigma(\mathcal{A})$ let $\mathcal{X}(S)$ and $\mathcal{X}_{(S)}$ denote the sets $\{x \in \mathcal{X} ; \operatorname{Sp}(x) \subseteq S\}$ and $\{x \in \mathcal{X} ; \operatorname{Sp}(x) \cap S=\emptyset\}$, respectively. Let $\mathcal{X}_{S}$ be the closure of $\mathcal{X}_{(S)}$. It is not hard to see that $\mathcal{X}(S)$ and $\mathcal{X}_{(S)}$ are linear sets. By use of Proposition 1.2(iii) we can prove that $\mathcal{X}(S)$ and $\mathcal{X}_{(S)}$ are $\mathcal{A}$-invariant. Hence $\mathcal{X}_{S}$ is an $\mathcal{A}$-submodule of $\mathcal{X}$. We have proven part (i) of the following proposition.

Proposition 2.7. Let $\mathcal{A}$ be a spectrally separable algebra.

(i) $\mathcal{X}_{S}$ is an $\mathcal{A}$-submodule for any subset $S \subseteq \Sigma(\mathcal{A})$.

(ii) If $F \subseteq \Sigma(\mathcal{A})$ is a compact subset, then $\mathcal{X}(F)$ is an $\mathcal{A}$-submodule of $\mathcal{X}$ and $\mathcal{X}^{*}(F)$ is a weak-* closed $\mathcal{A}$-submodule of the dual $\mathcal{A}$-module $\mathcal{X}^{*}$.

Proof. (ii) Let $F \subseteq \Sigma(\mathcal{A})$ be a compact subset and let $\left\{x_{n}\right\}_{n=1}^{\infty}$ be a sequence in $\mathcal{X}(F)$ such that $x_{n} \rightarrow x \in \mathcal{X}$. If $\varphi$ is in $F^{\mathrm{c}}$, then, by Lemma 2.6, there is $a \in \mathcal{A}$ such that $\widehat{a}(\varphi)=1$ and $\operatorname{Sp}(a) \subset F^{\mathrm{c}}$. Since $\mathcal{A}$ has a unit 
it follows from $\operatorname{Sp}\left(a x_{n}\right)=\emptyset$ that $a x_{n}=0$ for all $n=1,2, \ldots$ Hence

$$
\|a x\|=\left\|a x-a x_{n}\right\| \leq\|a\|\left\|x-x_{n}\right\| \rightarrow 0 \quad \text { as } n \rightarrow \infty,
$$

and thus $a \in \operatorname{ann}_{\mathcal{A}}(x)$. Hence $\varphi \notin \operatorname{Sp}(x)$ and consequently $x \in \mathcal{X}(F)$.

We can similarly see that $\mathcal{X}^{*}(F)$ is weak-* closed.

The modules $\mathcal{X}(F)$ and $\mathcal{X}_{S}$ are called spectral and co-spectral $\mathcal{A}$-submodules of $F$ and $S$, respectively (cf. [3]).

The following corollary of Proposition 2.7 can be easily proven with the help of Lemma 2.6.

Corollary 2.8. Let $\mathcal{A}$ be a spectrally separable algebra. Then $\mathcal{A}_{S}$ is a closed ideal in $\mathcal{A}$ for any subset $S$ of $\Sigma(\mathcal{A})$ and $\bar{S}=h\left(\mathcal{A}_{S}\right)$. If $F$ is a compact subset of $\Sigma(\mathcal{A})$, then $\mathcal{A}(F)$ is a closed ideal in $\mathcal{A}$ and its hull is $h(\mathcal{A}(F))=\overline{F^{\mathrm{c}}}$.

Now we can prove the following modification of Lemma 2.6.

Corollary 2.9. Let $\mathcal{A}$ be a spectrally separable algebra. Then for any two compact and disjoint subsets $E$ and $F$ of $\Sigma(\mathcal{A})$ there is $a \in \mathcal{A}$ such that $\widehat{a}(\tau)=1$ for all $\tau \in E$ and $\operatorname{Sp}(a) \cap F=\emptyset$.

Proof. Note first that every closed subset of $\Sigma(\mathcal{A})$ is compact because $\Sigma(\mathcal{A})$ is compact. Let $U$ and $V$ be disjoint open neighbourhoods of $E$ and $F$, respectively. Then $F \subset V \subset U^{\mathrm{c}}$ and $E \cap U^{\mathrm{c}}=\emptyset$. Since $\mathcal{A}$ is regular and $h\left(\mathcal{A}_{U^{\mathrm{c}}}\right)=U^{\mathrm{c}}$ (Corollary 2.8) we can use [10], Theorem 7.3.2: there is $a \in \mathcal{A}_{U^{\mathrm{c}}}$ such that $\widehat{a}(\tau)=1$ for all $\tau \in E$ and $\widehat{a}(\tau)=0$ for all $\tau \in U^{\mathrm{c}}$. Let us show that $\operatorname{Sp}(a) \cap F=\emptyset$. Indeed, if $\varphi \in \operatorname{Sp}(a) \cap F$ then $\varphi \notin V^{\mathrm{c}}$. By Lemma 2.6 there is $c \in \mathcal{A}$ such that $\widehat{c}(\varphi)=1$ and $\operatorname{Sp}(c) \cap V^{\mathrm{c}}=\emptyset$. If $b \in \mathcal{A}_{\left(U^{\mathrm{c}}\right)}$ then $\operatorname{Sp}(b) \cap U^{\mathrm{c}}=\emptyset$ and hence $\operatorname{Sp}(b) \subseteq V^{\mathrm{c}}$ because $U^{\mathrm{c}} \cup V^{\mathrm{c}}=\Sigma(\mathcal{A})$. Thus, $\mathcal{A}_{\left(U^{\mathrm{c}}\right)} \subseteq \mathcal{A}\left(V^{\mathrm{c}}\right)$ and therefore $\mathcal{A}_{U^{\mathrm{c}}} \subseteq \mathcal{A}\left(V^{\mathrm{c}}\right)$. It follows that $\operatorname{Sp}(a) \subseteq V^{\mathrm{c}}$. Now we have $\operatorname{Sp}(a) \cap \operatorname{Sp}(c)=\emptyset$ and hence $a c=0$, by Proposition 1.2. This contradicts the definition of $\operatorname{Sp}(a)$ because $\widehat{c}(\varphi)=1$ and $\varphi \in \operatorname{Sp}(a)$.

The following theorem is a very useful characterization of the spectrally separable algebras.

TheOrem 2.10 (Partition of unity, cf. [3]). Let $\mathcal{A}$ be a unital commutative Banach algebra. Then $\mathcal{A}$ is spectrally separable if and only if for any open covering $\mathcal{U}=\left\{U_{1}, \ldots, U_{n}\right\}$ of $\Sigma(\mathcal{A})$ there are $a_{1}, \ldots, a_{n}$ in $\mathcal{A}$ such that $a_{1}+\ldots+a_{n}=1$ and $\operatorname{Sp}\left(a_{k}\right) \subset U_{k}$ for all $k=1, \ldots, n$.

Proof. For every $\varphi \in \Sigma(\mathcal{A})$ there is $U_{i}$ in the covering $\mathcal{U}$ such that $\varphi \in U_{i}$. Since $\Sigma(\mathcal{A})$ is a Hausdorff topological space, an open neighbourhood $W_{\varphi}$ of $\varphi$ can be found such that $W_{\varphi} \subset \bar{W}_{\varphi} \subset U_{i}$. The family $\left\{W_{\varphi} ; \varphi \in \Sigma(\mathcal{A})\right\}$ is an open covering of $\Sigma(\mathcal{A})$ and since $\Sigma(\mathcal{A})$ is compact there are $\varphi_{1}, \ldots, \varphi_{m}$ in $\Sigma(\mathcal{A})$ such that $\Sigma(\mathcal{A})=W_{\varphi_{1}} \cup \ldots \cup W_{\varphi_{m}}$. Let $E_{i}$ be the union of those $\bar{W}_{\varphi_{j}}$ which are subsets of $U_{i}$. Thus, for every index $i$ the set $E_{i}$ is a compact 
subset of $U_{i}$ and $E_{1} \cup \ldots \cup E_{n}=\Sigma(\mathcal{A})$. By Corollary 2.9 there are $c_{i} \in \mathcal{A}$, $i=1, \ldots, n$, such that $\widehat{c}_{i}(\tau)=1$ for all $\tau \in E_{i}$ and $\operatorname{Sp}\left(c_{i}\right) \cap U_{i}^{\mathrm{c}}=\emptyset$. Set

$$
b_{1}=c_{1}, b_{2}=\left(1-c_{1}\right) c_{2}, \ldots, b_{n}=\left(1-c_{1}\right)\left(1-c_{2}\right) \ldots\left(1-c_{n-1}\right) c_{n} .
$$

Then $\operatorname{Sp}\left(b_{i}\right) \subseteq \operatorname{Sp}\left(c_{i}\right) \subset U_{i}$ and it is easily seen by induction that $b_{1}+\ldots+b_{k}$ $=1-\left(1-c_{1}\right) \ldots\left(1-c_{k}\right)$. Thus, we have $b_{1}+\ldots+b_{n}=1-\left(1-c_{1}\right) \ldots\left(1-c_{n}\right)$. Since every $\varphi \in \Sigma(\mathcal{A})$ is in some $E_{i}$, we have $\varphi\left(1-c_{i}\right)=0$. It follows that $c=\left(1-c_{1}\right) \ldots\left(1-c_{n}\right)$ is in the radical of $\mathcal{A}$. Thus, $1-c$ is invertible. If we set $a_{i}=b_{i}(1-c)^{-1}$ for all $i=1, \ldots, n$, then $a_{1}+\ldots+a_{n}$ $=1$ and $\operatorname{Sp}\left(a_{i}\right) \subset U_{i}$.

It is clear (see the second part of the proof of Lemma 2.6) that a unital commutative Banach algebra is spectrally separable if for any open covering $\mathcal{U}=\left\{U_{1}, \ldots, U_{n}\right\}$ of $\Sigma(\mathcal{A})$ there exist $a_{1}, \ldots, a_{n}$ in $\mathcal{A}$ such that $a_{1}+\ldots+a_{n}$ $=1$ and $\operatorname{Sp}\left(a_{k}\right) \subset U_{k}(k=1, \ldots, n)$.

3. Multiplication operators. In this section we characterize the spectrally separable algebras by means of multiplication operators. We first recall some basic notions from the axiomatic spectral theory of linear operators on Banach spaces. The reader is referred to [14], especially to Chapters 1 and 4 of this excellent monograph, for details.

A bounded linear operator $T$ on a complex Banach space $\mathcal{X}$ is decomposable if for every open covering $\{U, V\}$ of the complex plane there are closed subspaces $\mathcal{Y}$ and $\mathcal{Z}$ of $\mathcal{X}$ which are invariant for $T$, their sum is $\mathcal{X}$, and the spectrum $\sigma(T)$ of $T$ splits in the sense that $\sigma\left(\left.T\right|_{\mathcal{Y}}\right) \subset U$ and $\sigma\left(\left.T\right|_{\mathcal{Z}}\right) \subset V$.

We shall also need the following closely related notions. An operator $T \in B(\mathcal{X})$ is said to have Bishop's property $(\beta)$ if for every open subset $U$ of $\mathbb{C}$ and for every sequence of analytic functions $f_{n}: U \rightarrow \mathcal{X}$ for which $(T-\lambda) f_{n}(\lambda)$ converges uniformly to zero on each compact subset of $U$, we have $f_{n}(\lambda) \rightarrow 0$ as $n \rightarrow \infty$, uniformly on each compact subset of $U$. Property $(\beta)$ implies that $T$ has the single-valued extension property (SVEP), which means that for every open $U \subseteq \mathbb{C}$ the only analytic solution $f: U \rightarrow \mathcal{X}$ of the equation $(T-\lambda) f(\lambda)=0$ for all $\lambda \in U$ is the constant $f \equiv 0$. Finally, an operator $T \in B(\mathcal{X})$ has the decomposition property $(\delta)$ if for an arbitrary open covering $\left\{U_{1}, U_{2}\right\}$ of $\mathbb{C}$ every $x \in \mathcal{X}$ admits a decomposition $x=u_{1}+u_{2}$ where the vectors $u_{1}$ and $u_{2}$ satisfy

$$
u_{k}=(T-\lambda) f_{k}(\lambda) \quad \text { for all } \lambda \in \mathbb{C} \backslash \bar{U}_{k}
$$

and some analytic functions $f_{k}: \mathbb{C} \backslash \bar{U}_{k} \rightarrow \mathcal{X}, k=1,2$.

An operator $T \in B(\mathcal{X})$ is decomposable if and only if it has both properties $(\beta)$ and $(\delta)$. It has been shown in [1] that the properties $(\beta)$ and $(\delta)$ are dual to each other: the operator $T$ satisfies $(\beta)$ if and only if the adjoint 
operator $T^{*}$ on the dual space $\mathcal{X}^{*}$ satisfies $(\delta)$, and the statement remains valid if the properties are interchanged.

If $\mathcal{S}$ is any family of bounded linear operators on a Banach space $\mathcal{X}$ we will denote by Lat $\mathcal{S}$ the family of all closed subspaces of $\mathcal{X}$ which are invariant for all operators in $\mathcal{S}$. Let $\operatorname{cl}(\mathbb{C})$ be the family of all closed subsets of the complex plane.

Definition 3.1. Let $T$ be a bounded linear operator on a Banach space $\mathcal{X}$. A mapping $\mathrm{E}: \operatorname{cl}(\mathbb{C}) \rightarrow \operatorname{Lat}\{T\}$ is a spectral capacity for $T$ if the following conditions are fulfilled:

(a) $\mathrm{E}(\emptyset)=\{0\}$ and $\mathrm{E}(\mathbb{C})=\mathcal{X}$;

(b) $\mathcal{X}=\mathrm{E}\left(\bar{U}_{1}\right)+\ldots+\mathrm{E}\left(\bar{U}_{n}\right)$ for every finite open cover $\left\{U_{1}, \ldots, U_{n}\right\}$ of $\mathbb{C}$;

(c) $\mathrm{E}\left(\bigcap_{n=1}^{\infty} F_{n}\right)=\bigcap_{n=1}^{\infty} \mathrm{E}\left(F_{n}\right)$ for every countable family of closed sets $F_{n} \subseteq \mathbb{C}$;

(d) $\sigma\left(\left.T\right|_{\mathrm{E}(F)}\right) \subseteq F$ for every closed set $F \subseteq \mathbb{C}$.

Let $T$ be a bounded linear operator on a Banach space $\mathcal{X}$. Then the local resolvent set $\varrho_{T}(x)$ of $T$ at the point $x \in \mathcal{X}$ is defined as the union of all open subsets $U$ of $\mathbb{C}$ for which there is an analytic function $f: U \rightarrow \mathcal{X}$ such that

$$
(T-\lambda) f(\lambda)=x \quad \text { for all } \lambda \in U .
$$

The local spectrum $\sigma_{T}(x)$ of $T$ at $x$ is then defined as $\sigma_{T}(x):=\mathbb{C} \backslash \varrho_{T}(x)$. Evidently, $\varrho_{T}(x)$ is open and $\sigma_{T}(x)$ is closed. The resolvent set $\varrho(T)$ is always a subset of $\varrho_{T}(x)$, hence $\sigma_{T}(x)$ is a subset of the spectrum $\sigma(T)$ of $T$.

We define the analytic spectral subspaces of $T$ by

$$
X_{T}(M):=\left\{x \in \mathcal{X} ; \sigma_{T}(x) \subseteq M\right\}
$$

for all subsets $M \subseteq \mathbb{C}$. It is clear that $X_{T}(M)=X_{T}(M \cap \sigma(T))$ and $X_{T}(M) \subseteq X_{T}(N)$ whenever $M \subseteq N \subseteq \mathbb{C}$. In fact, it follows immediately from the definition that

$$
X_{T}\left(\bigcap_{i \in I} M_{i}\right)=\bigcap_{i \in I} X_{T}\left(M_{i}\right)
$$

for every family of sets $M_{i} \subseteq \mathbb{C}$.

Every analytic spectral subspace $X_{T}(M)$ of $T$ is a linear subspace of $\mathcal{X}$ but in general it is not closed. It is well known that a bounded linear operator $T$ on a Banach space $\mathcal{X}$ is decomposable if and only if there is a spectral capacity for $T$. Moreover, if the operator $T$ is decomposable, then for every closed $F \subseteq \mathbb{C}$ the corresponding analytic spectral subspace $X_{T}(F)$ is closed, and the spectral capacity of the operator $T$ is uniquely determined and given by $\mathrm{E}(F)=X_{T}(F)$ for all closed subsets $F \subseteq \mathbb{C}$ (see [14], Theorem 1.2.23). 
The following theorem is an extension of a part of Theorem 2 of [3].

TheOrem 3.2. Let $\mathcal{A}$ be a spectrally separable algebra and let $\mathcal{X}$ be a Banach left $\mathcal{A}$-module. Then every multiplication operator $T_{a}: \mathcal{X} \rightarrow \mathcal{X}, x \mapsto$ $a x, a \in \mathcal{A}, x \in \mathcal{X}$, has a spectral capacity. Thus, every $T_{a}$ is decomposable.

Proof. Let $a \in \mathcal{A}$. The Gelfand transform $\widehat{a}$ of $a$ is a continuous function. Hence $\widehat{a}^{-1}(M)$ is an open (resp. closed) subset of $\Sigma(\mathcal{A})$ if $M$ is an open (resp. closed) subset of $\mathbb{C}$. Thus, if $F \subseteq \mathbb{C}$ is closed then $\widehat{a}^{-1}(F)$ is a compact subset of $\Sigma(\mathcal{A})$ and, by Proposition 2.7, $\mathcal{X}\left(\widehat{a}^{-1}(F)\right)$ is an $\mathcal{A}$-submodule of $\mathcal{X}$. Define a mapping $\mathrm{E}: \operatorname{cl}(\mathbb{C}) \rightarrow \operatorname{Lat}\left\{T_{a}\right\}$ by $\mathrm{E}(F)=\mathcal{X}\left(\widehat{a}^{-1}(F)\right)$. We will show that $\mathrm{E}$ is a spectral capacity for $T_{a}$.

Clearly, $\mathrm{E}(\emptyset)=\mathcal{X}(\emptyset)=\{0\}$ and $\mathrm{E}(\mathbb{C})=\mathcal{X}(\Sigma(\mathcal{A}))=\mathcal{X}$. It is also quite standard to see that the condition (c) of Definition 3.1 is fulfilled.

Let $\left\{U_{1}, \ldots, U_{n}\right\}$ be an open covering of the complex plane $\mathbb{C}$. Then $\left\{\mathcal{U}_{1}, \ldots, \mathcal{U}_{n}\right\}, \mathcal{U}_{k}=\widehat{a}^{-1}\left(U_{k}\right)$, is an open covering of $\Sigma(\mathcal{A})$. Of course, for an open subset $U \subseteq \mathbb{C}$ and $\mathcal{U}=\widehat{a}^{-1}(U)$ the closure $\overline{\mathcal{U}}$ is a subset of $\widehat{a}^{-1}(\bar{U})$. Thus, $\mathcal{X}\left(\overline{\mathcal{U}}_{k}\right) \subseteq \mathcal{X}\left(\widehat{a}^{-1}\left(\bar{U}_{k}\right)\right)=\mathrm{E}\left(\bar{U}_{k}\right)$. The inclusion $\mathrm{E}\left(\bar{U}_{1}\right)+\ldots+\mathrm{E}\left(\bar{U}_{n}\right) \subseteq \mathcal{X}$ is clear. Let $x \in \mathcal{X}$. By Theorem 2.10 there are $c_{k}$ in $\mathcal{A}, k=1, \ldots, n$, such that $c_{1}+\ldots+c_{n}=1$ and $\operatorname{Sp}\left(c_{k}\right) \subseteq \mathcal{U}_{k}, k=1, \ldots, n$. Hence $x=c_{1} x+\ldots+c_{n} x$ and $\operatorname{Sp}\left(c_{k} x\right) \subseteq \mathcal{U}_{k} \subseteq \overline{\mathcal{U}}_{k}, k=1, \ldots, n$. It follows $x \in \mathrm{E}\left(\bar{U}_{1}\right)+\ldots+\mathrm{E}\left(\bar{U}_{n}\right)$.

Let $F$ be a closed subset of $\mathbb{C}$ and define $\mathcal{F}=\widehat{a}^{-1}(F)$. If $\lambda$ is any point in $F^{\text {c }}$ then there is an open neighbourhood $U$ of $F$ such that $\lambda \notin \bar{U}$. We have mentioned that $\overline{\mathcal{U}} \subseteq \widehat{a}^{-1}(\bar{U})$, where $\mathcal{U}=\widehat{a}^{-1}(U)$. Set $H=U^{\mathrm{c}}$ and $\mathcal{H}=\widehat{a}^{-1}(H)$. The sets $H$ and $\mathcal{H}$ are closed. It is evident that $\mathcal{F} \cap \mathcal{H}=\emptyset$. Since $\mathcal{H}^{\mathrm{c}}=\widehat{a}^{-1}\left(H^{\mathrm{c}}\right)$ and $H^{\mathrm{c}}=U$ we have $\overline{\mathcal{H}^{\mathrm{c}}} \subseteq \widehat{a}^{-1}(\bar{U})$. Hence the spectrum of the quotient algebra $\mathcal{A} / \mathcal{A}(\mathcal{H})$ is contained in $\widehat{a}^{-1}(\bar{U})$, because $\Sigma(\mathcal{A} / \mathcal{A}(\mathcal{H}))=$ $h(\mathcal{A}(\mathcal{H}))=\overline{\mathcal{H}^{\mathrm{c}}}$ by [10], Theorem 7.3.1. Consider the element $a-\lambda+\mathcal{A}(\mathcal{H}) \in$ $\mathcal{A} / \mathcal{A}(\mathcal{H})$. For each $\varphi \in \Sigma(\mathcal{A} / \mathcal{A}(\mathcal{H}))$ we have $\varphi(a-\lambda+\mathcal{A}(\mathcal{H}))=\widehat{a}(\varphi)-\lambda$. Since $\varphi$ is not in $\bar{U}$ it follows that $\widehat{a}(\varphi) \neq \lambda$. Thus, zero is not in the spectrum $\sigma(a-\lambda+\mathcal{A}(\mathcal{H}))$ or, equivalently, $a-\lambda+\mathcal{A}(\mathcal{H})$ has an inverse in $\mathcal{A} / \mathcal{A}(\mathcal{H})$, that is, there exists $b \in \mathcal{A}$ such that $(b+\mathcal{A}(\mathcal{H}))(a-\lambda+\mathcal{A}(\mathcal{H}))=1+\mathcal{A}(\mathcal{H})$. Let $d \in \mathcal{A}(\mathcal{H})$ be such that $b(a-\lambda)=1+d$. For every $x \in \mathcal{X}(\mathcal{F})$ we have

$$
\left.T_{b}\right|_{\mathcal{X}(\mathcal{F})}\left(\left.T_{a}\right|_{\mathcal{X}(\mathcal{F})}-\lambda\right) x=b(a-\lambda) x=x+d x .
$$

Since $\operatorname{Sp}(d x) \subseteq \operatorname{Sp}(d) \cap \operatorname{Sp}(x) \subseteq \mathcal{H} \cap \mathcal{F}=\emptyset$, the vector $d x$ is zero and hence

$$
\left.T_{b}\right|_{\mathcal{X}(\mathcal{F})}\left(\left.T_{a}\right|_{\mathcal{X}(\mathcal{F})}-\lambda\right) x=x, \quad x \in \mathcal{X}(\mathcal{F}) .
$$

Thus, $\left.T_{a}\right|_{\mathcal{X}(\mathcal{F})}-\lambda$ is invertible and it follows that $\sigma\left(\left.T_{a}\right|_{\mathcal{X}(\mathcal{F})}\right) \subseteq F$.

Definition 3.3. A bounded linear operator $T$ on a Banach space $\mathcal{X}$ is super-decomposable if for every pair of open sets $U, V \subseteq \mathbb{C}$ which cover $\mathbb{C}$, 
there exists $S \in B(\mathcal{X})$ such that $S T=T S, \sigma\left(\left.T\right|_{\overline{\operatorname{im} S}}\right) \subset U$ and $\sigma\left(\left.T\right|_{\overline{\operatorname{im}(I-S)}}\right)$ $\subset V$.

The notion of super-decomposable operators was introduced in [11] by Laursen and Neumann. They proved that an operator $T \in B(\mathcal{X})$ is superdecomposable if and only if for every open covering $\left\{U_{1}, U_{2}\right\}$ of $\mathbb{C}$ there are subspaces $\mathcal{X}_{1}, \mathcal{X}_{2} \in \operatorname{Lat}\{T\}$ as well as operators $S_{1}, S_{2} \in B(\mathcal{X})$, commuting with $T$, such that $S_{1}+S_{2}=I, S_{k}(\mathcal{X}) \subseteq \mathcal{X}_{k}, k=1,2$ and $\sigma\left(\left.T\right|_{\mathcal{X}_{k}}\right) \subset U_{k}$, $k=1,2$ (see [11], Theorem 1.4). Since every super-decomposable operator is decomposable (cf. [11], Theorem 1.3), the following theorem is an improvement of Theorem 3.2 and hence of Theorem 2 in [3].

THEOREM 3.4. Let $\mathcal{A}$ be a spectrally separable algebra and let $\mathcal{X}$ be a Banach left $\mathcal{A}$-module. Then the multiplication operator $T_{a}: x \mapsto a x, x \in \mathcal{X}$, is super-decomposable for every $a \in \mathcal{A}$.

Proof. Let $a \in \mathcal{A}$. If $\left\{W_{1}, W_{2}\right\}$ is an open covering of $\mathbb{C}$ let $\left\{U_{1}, U_{2}\right\}$ be an open covering of $\mathbb{C}$ such that $U_{k} \subset \bar{U}_{k} \subset W_{k}, k=1,2$. The pair $\left\{\mathcal{U}_{1}, \mathcal{U}_{2}\right\}$, where $\mathcal{U}_{k}=\widehat{a}^{-1}\left(U_{k}\right), \quad k=1,2$, is an open covering of $\Sigma(\mathcal{A})$. Hence, by Theorem 2.10, there are $b_{1}$ and $b_{2}$ in $\mathcal{A}$ such that $b_{1}+b_{2}=1$ and $\operatorname{Sp}\left(b_{k}\right) \subset$ $\mathcal{U}_{k}, k=1,2$. The operators $S_{k}=T_{b_{k}}, k=1,2$, commute with $T_{a}$ and $S_{1}+S_{2}=I$. According to the proof of Theorem 3.2 the spectral subspaces $\mathcal{X}_{k}=\mathcal{X}\left(\widehat{a}^{-1}\left(\bar{U}_{k}\right)\right), k=1,2$, are in $\operatorname{Lat}\left\{T_{a}\right\}$ and $\sigma\left(T_{a} \mid \mathcal{X}_{k}\right) \subseteq \bar{U}_{k} \subset W_{k}$, $k=1,2$. Hence for every $x \in \mathcal{X}$ we have

$$
\operatorname{Sp}\left(S_{k} x\right)=\operatorname{Sp}\left(b_{k} x\right) \subseteq \operatorname{Sp}\left(b_{k}\right) \subset \overline{\mathcal{U}}_{k} \subseteq \widehat{a}^{-1}\left(\bar{U}_{k}\right), \quad k=1,2 .
$$

Thus, $\overline{\operatorname{im} S_{k}} \subseteq \mathcal{X}_{k}, k=1,2$.

The following theorem improves Theorem 2 of [3] in the opposite direction.

TheOREM 3.5. Let $\mathcal{A}$ be a unital commutative Banach algebra. If there is a subset $\mathcal{A}_{0}$ in $\mathcal{A}$ such that the Gelfand transforms of elements in $\mathcal{A}_{0}$ separate points of the spectrum $\Sigma(\mathcal{A})$ and for every $a \in \mathcal{A}_{0}$ the multiplication operator $T_{a}: \mathcal{A} \rightarrow \mathcal{A}$ has the decomposition property $(\delta)$, then $\mathcal{A}$ is a spectrally separable algebra.

Proof. Let $\varphi$ and $\psi$ be two distinct functionals in $\Sigma(\mathcal{A})$. Then there is $a \in \mathcal{A}_{0}$ such that $\lambda_{0}=\widehat{a}(\varphi) \neq \widehat{a}(\psi)=\mu_{0}$. Define $\varepsilon=\left|\lambda_{0}-\mu_{0}\right|$ and let $U=\left\{z \in \mathbb{C} ;\left|\lambda_{0}-z\right|<\varepsilon / 3\right\}, V=\left\{z \in \mathbb{C} ;\left|\mu_{0}-z\right|<\varepsilon / 3\right\}, W=\{z \in$ $\mathbb{C} ;\left|\lambda_{0}-z\right|>\varepsilon / 4$ and $\left.\left|\mu_{0}-z\right|>\varepsilon / 4\right\}$, and $U_{1}=V \cup W, V_{1}=U \cup W$. Since $T_{a}$ has the decomposition property $(\delta)$ and $\left\{U, U_{1}\right\}$ is an open covering of $\mathbb{C}$, every $x \in \mathcal{A}$ admits a decomposition $x=u+u_{1}$ where $u=\left(T_{a}-\lambda\right) f(\lambda)$ for all $\lambda \in \mathbb{C} \backslash \bar{U}$ and an analytic function $f: \mathbb{C} \backslash \bar{U} \rightarrow \mathcal{A}$ and similarly $u_{1}=\left(T_{a}-\lambda\right) f_{1}(\lambda)$ for all $\lambda \in \mathbb{C} \backslash \bar{U}_{1}$ and an analytic function $f_{1}: \mathbb{C} \backslash \bar{U}_{1} \rightarrow \mathcal{A}$. It follows that $\sigma_{T_{a}}(u) \subseteq \bar{U}$ and $\sigma_{T_{a}}\left(u_{1}\right) \subseteq \bar{U}_{1}$. Of course, $x \in \mathcal{A}$ also admits 
a decompostion $x=v+v_{1}$ where $v$ and $v_{1}$ have similar properties with respect to $V, V_{1}$.

For $x=1$ we have $1=u+\left(T_{a}-\lambda\right) f_{1}(\lambda), \lambda \in \mathbb{C} \backslash \bar{U}_{1}$. Since $\lambda_{0} \in \mathbb{C} \backslash \bar{U}_{1}$, it follows from $1=u+\left(a-\lambda_{0}\right) f_{1}\left(\lambda_{0}\right)$ that $\widehat{u}(\varphi)=1$. In the same way we can see that $\widehat{v}(\psi)=1$. Hence, it remains to prove that $u v=0$.

Let $\xi \in \mathcal{A}^{*}$. Consider $\mathcal{A}^{*}$ as a dual Banach left $\mathcal{A}$-module. Since $f$ and $f_{1}$ are analytic $\mathcal{A}$-valued functions, the $\mathcal{A}^{*}$-valued functions $F(\lambda)=f(\lambda) \xi$, $\lambda \in \mathbb{C} \backslash \bar{U}$, and $F_{1}(\lambda)=f_{1}(\lambda) \xi, \lambda \in \mathbb{C} \backslash \bar{U}_{1}$, are also analytic. We have

$$
u \xi=\left(T_{a}-\lambda\right) f(\lambda) \xi=(a-\lambda) f(\lambda) \xi=\left(T_{a}^{*}-\lambda\right) F(\lambda), \quad \lambda \in \mathbb{C} \backslash \bar{U},
$$

and $u_{1} \xi=\left(T_{a}^{*}-\lambda\right) F_{1}(\lambda), \lambda \in \mathbb{C} \backslash \bar{U}_{1}$, so $\sigma_{T_{a}^{*}}(u \xi) \subseteq \bar{U}$. Similarly, $\sigma_{T_{a}^{*}}(v \xi) \subseteq$ $\bar{V}$. Denote by $H$ the function $\lambda \mapsto v F(\lambda), \lambda \in \mathbb{C} \backslash \bar{U}$. Since $H$ is analytic, $\sigma_{T_{a}^{*}}(v u \xi) \subseteq \bar{U}$. Similarly, $\sigma_{T_{a}^{*}}(v u \xi) \subseteq \bar{V}$. Hence, $\sigma_{T_{a}^{*}}(v u \xi) \subseteq \bar{U} \cap \bar{V}=\emptyset$. The operator $T_{a}$ has property $(\delta)$, so the adjoint operator $T_{a}^{*}$ has Bishop's property $(\beta)$ and thus has SVEP or, equivalently, $\mathcal{A}_{T_{a} *}^{*}(\emptyset)=\{0\}$. Therefore $v u \xi=0$ and we have $0=\langle v u \xi, 1\rangle=\langle\xi, u v\rangle$ for all $\xi \in \mathcal{A}^{*}$. Thus, $u v=0$.

EXAMPLE 3.6. Let $\mathcal{X}$ be a Banach space and $B(\mathcal{X})$ the algebra of all bounded linear operators on $\mathcal{X}$. Let $T \in B(\mathcal{X})$ be a generalized scalar operator with a spectral distribution $\mathrm{U}: C^{\infty}\left(\mathbb{R}^{2}\right) \rightarrow B(\mathcal{X})$ (see [5], Chapter 4). Denote by $\mathcal{A}$ the uniform closure of $\left\{\mathrm{U}(f) ; f \in C^{\infty}\left(\mathbb{R}^{2}\right)\right\}$. Then $\mathcal{A}$ is a commutative Banach subalgebra in $B(\mathcal{X})$ and the identity operator $I$ is in $\mathcal{A}$. The spectrum $\Sigma(\mathcal{A})$ can be identified with $\sigma(T)$ and the Gelfand transform of $\mathrm{U}(f)$ is $\left.f\right|_{\sigma(T)}$ (cf. [5], Theorem 3.2.1).

Following the proof of Theorem 2.13 in [2] we can see that the multiplication operators $L_{U(f)}: \mathcal{A} \rightarrow \mathcal{A}, f \in C^{\infty}\left(\mathbb{R}^{2}\right)$, are decomposable. Hence the algebra is spectrally separable because the $\left.f\right|_{\sigma(T)}$ for $f \in C^{\infty}\left(\mathbb{R}^{2}\right)$ separate points of $\sigma(T)$.

Let $\mathcal{X}$ and $\mathcal{Y}$ be Banach spaces and let $T \in B(\mathcal{X})$ and $S \in B(\mathcal{Y})$. If $T$ has property $(\delta)$ and if there is a surjection $\pi \in B(\mathcal{X}, \mathcal{Y})$ such that $\pi T=S \pi$, then also $S$ has property $(\delta)$ ([1], Lemma 2). We will use this fact in the proof of the last theorem of this article.

Theorem 3.7. Let $\mathcal{A}$ be a unital commutative Banach algebra and let $\mathcal{X}$ be a left Banach $\mathcal{A}$-module. Assume that $\mathcal{A}$ acts cyclically on $\mathcal{X}$ and that $\operatorname{ann}_{\mathcal{A}}(\mathcal{X})=\{0\}$. If there is a subset $\mathcal{A}_{0}$ in $\mathcal{A}$ such that the Gelfand transforms of elements in $\mathcal{A}_{0}$ separate points of the spectrum $\Sigma(\mathcal{A})$ and for every $a \in \mathcal{A}_{0}$ the multiplication operator $L_{a}: \mathcal{X} \rightarrow \mathcal{X}$ has the decomposition property $(\delta)$, then $\mathcal{A}$ is a spectrally separable algebra.

Proof. Let $x \in \mathcal{X}$ be cyclic for $\mathcal{A}$, i.e. $\{a x ; a \in \mathcal{A}\}=\mathcal{X}$. It is clear that the mapping $\pi: a \mapsto a x$ is a well defined surjection and that it is in $B(\mathcal{A}, \mathcal{X})$. If $a x=0$ for some $a \in \mathcal{A}$, then $a b x=0$ for all $b \in \mathcal{A}$. Since $x$ is cyclic and the annihilator of $\mathcal{X}$ is trivial it follows that $\pi$ is injective. Hence 
$\pi^{-1}: \mathcal{X} \rightarrow \mathcal{A}$ is a continuous linear map. Let $a$ be in $\mathcal{A}_{0}$ and let $T_{a}$ and $L_{a}$ be the corresponding multiplication operators on $\mathcal{A}$, respectively on $\mathcal{X}$. Then $T_{a}$ has property $(\delta)$ because $\pi^{-1} L_{a}=T_{a} \pi^{-1}$ and $L_{a}$ has property $(\delta)$. Now Theorem 3.5 can be used.

The condition $\operatorname{ann}_{\mathcal{A}}(\mathcal{X})=\{0\}$ cannot be removed from Theorem 3.7. Let $\mathcal{A}$ be the disc algebra, i.e. the Banach algebra of all continuous complexvalued functions on the closed unit disc $\overline{\mathbb{D}}$ which are analytic on the open unit disc $\mathbb{D}$. Since $\mathcal{A}$ is not regular it is also not spectrally separable. The one-dimensional space $\mathbb{C}$ is a left $\mathcal{A}$-module if the multiplication is defined by $f z:=f(0) z$ for each $f \in \mathcal{A}$ and each $z \in \mathbb{C}$. Of course, $\mathcal{A}$ acts cyclically on $\mathbb{C}$ and the multiplication operator corresponding to $f \in \mathcal{A}$ is $f(0) I$, where $I$ is the identity operator on $\mathbb{C}$. Thus, all conditions of Theorem 3.7 are fulfilled, except that $\operatorname{ann}_{\mathcal{A}}(\mathbb{C}) \neq\{0\}$.

The author does not know if Theorem 3.7 is valid when the cyclic action of $\mathcal{A}$ on $\mathcal{X}$ is replaced by a weaker condition. However, the following example shows that some additional condition must be present. Let $\mathcal{A}$ again be the disc algebra and let $\mathcal{X}$ be the Banach algebra of all continuous complexvalued fuctions defined on $\overline{\mathbb{D}}$. Then each multiplication operator on $\mathcal{X}$ by an element from $\mathcal{A}$ is super-decomposable because $\mathcal{A} \subset \mathcal{X}$ and $\mathcal{X}$ is spectrally separable. We also have $\operatorname{ann}_{\mathcal{A}}(\mathcal{X})=\{0\}$. However, $\mathcal{A}$ does not act cyclically on $\mathcal{X}$.

Acknowledgements. The author would like to express his thanks to the referee and to Professor Milan Hladnik for their several helpful comments and suggestions.

\section{References}

[1] E. Albrecht and J. Eschmeier, Analytic functional models and local spectral theory, Proc. London Math. Soc. (3) 75 (1997), 323-348.

[2] C. Apostol, Decomposable multiplication operators, Rev. Roumaine Math. Pures Appl. 17 (1972), 323-333.

[3] A. G. Baskakov, Spectral synthesis in Banach modules over commutative Banach algebras, Math. Notes 34 (1983), 776-782 (transl. from Mat. Zametki).

[4] F. F. Bonsall and J. Duncan, Complete Normed Algebras, Springer, 1973.

[5] I. Colojoară and C. Foiaş, Theory of Generalized Spectral Operators, Gordon and Breach, New York, 1968.

[6] J. Eschmeier, K. B. Laursen and M. M. Neumann, Multipliers with natural local spectra on commutative Banach algebra, J. Funct. Anal. 138 (1996), 273-294.

[7] Şt. Frunză, A characterization of regular Banach algebras, Rev. Roumaine Math. Pures Appl. 18 (1973), 1057-1059.

[8] E. Hewitt and K. A. Ross, Abstract Harmonic Analysis, I, Springer, 1963.

[9] K. Koh, On a representation of a strongly harmonic ring by sheaves, Pacific J. Math. 41 (1972), 459-468. 
[10] R. Larsen, Banach Algebras, Dekker, 1973.

[11] K. B. Laursen and M. M. Neumann, Decomposable operators and automatic continuity, J. Operator Theory 15 (1986), 33-51.

[12] - - - Local spectral properties of multipliers on Banach algebras, Arch. Math. (Basel) 58 (1992), 368-375.

[13] - - -, Decomposable multipliers and applications to harmonic analysis, Studia Math. 101 (1992), 193-214.

[14] - - -, An Introduction to Local Spectral Theory, London Math. Soc. Monographs 20, Clarendon Press, Oxford, 2000.

[15] M. M. Neumann, Commutative Banach algebras and decomposable operators, Monatsh. Math. 113 (1992), 227-243.

[16] - Banach algebras, decomposable convolution operators, and a spectral mapping property, in: Function Spaces (Edwardsville, IL, 1990), Dekker, New York, 1991, 307-323.

[17] T. W. Palmer, Banach Algebras and the General Theory of *-Algebras, Volume I: Algebras and Banach Algebras, Cambridge Univ. Press, 1994.

[18] S. Teleman, Analyse harmonique dans les algèbres régulières, Rev. Roumaine Math. Pures Appl. 13 (1968), 691-750.

[19] F.-H. Vasilescu, Analytic Functional Calculus and Spectral Decomposition, Editura Academiei, Bucureşti, and Reidel, Dordrecht, 1982.

\section{IMFM}

University of Ljubljana

Jadranska 19

1111 Ljubljana, Slovenia

E-mail: janko.bracic@guest.arnes.si

Received August 14, 2000

Revised version September 4, 2001 